\title{
Turning Direction Determination using Error Dynamics-Based Guidance Law for Stationary Target Observation
}

\author{
By Mingu KIM and Youdan KIM \\ School of Mechanical and Aerospace Engineering, The Institute of Advanced Aerospace Technology, \\ Seoul National University, Seoul, Republic of Korea
}

(Received September 13th, 2012)

\begin{abstract}
Guidance laws are proposed to enable unmanned aerial vehicles (UAVs) to accomplish stationary target observation missions. In this paper, a nonlinear guidance law is designed utilizing the characteristics of distance error dynamics. The turning direction of the UAVs is very important for multiple UAVs operation. The turning direction of UAV is related to the line-of-sight angular acceleration of the UAV. The proposed guidance law is analyzed to provide a guideline for selecting guidance gains taking into consideration the turning direction. Numerical simulations are performed to verify the performance of the proposed guidance law.
\end{abstract}

Key Words: Guidance Law, Stationary Target Observation, Turning Direction, Multiple UAVs

\section{Nomenclature}

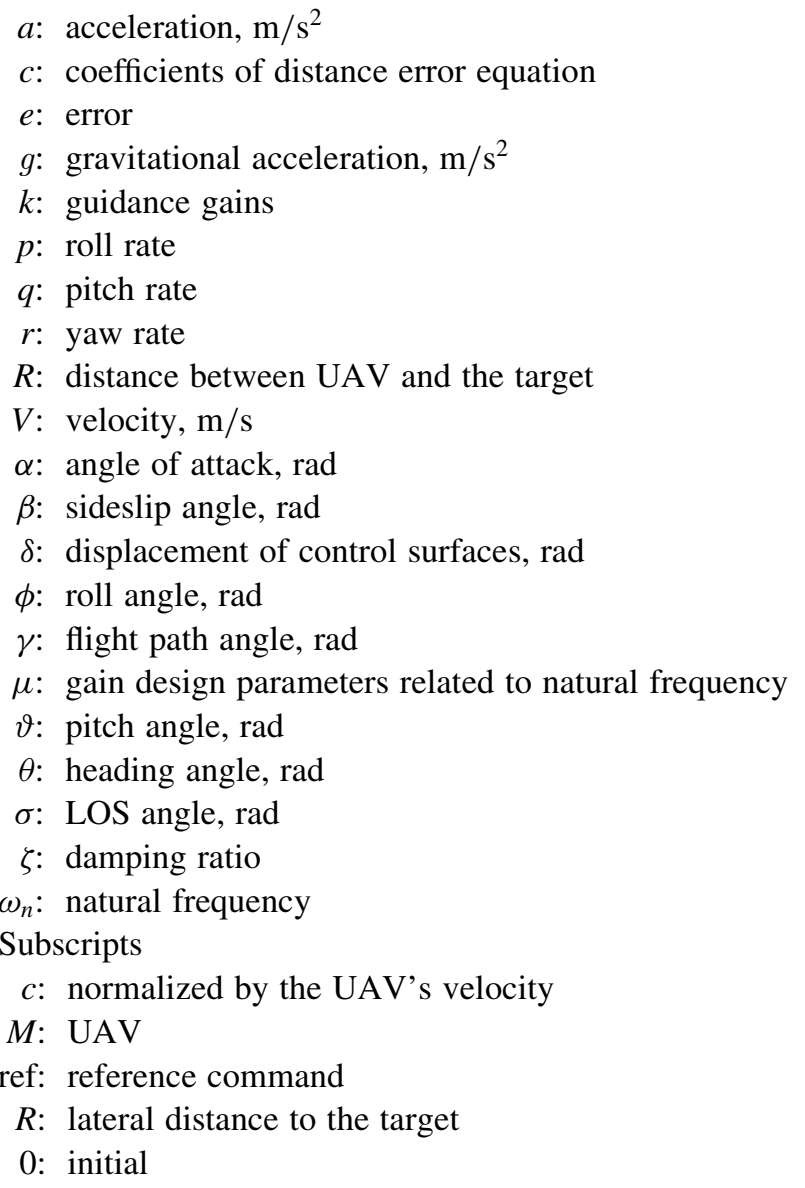

\section{Introduction}

A lot of research on the guidance of unmanned aerial vehicle (UAV) systems has been done, since the guidance system is very important for the UAV to track its designed trajectory. Multiple UAVs are very effective in accomplish-

(C) 2014 The Japan Society for Aeronautical and Space Sciences ing complicated military missions including surveillance, aerial reconnaissance and observation. Therefore, various nonlinear guidance laws for multiple UAVs have been developed.

In the last decade, vector field guidance has been applied to autonomous UAV missions, especially for standoff tracking of targets. ${ }^{1-3)}$ Lyapunov vector field guidance was constructed for multiple UAVs to perform a coordinated standoff tracking mission. ${ }^{1)}$ The guidance vector fields produce a stable convergence to a circling limit cycle behavior. ${ }^{2)}$ The produced vector fields guarantee global stability for the multiple UAVs. Summers et al. used the Lyapunov vector field approach to achieve the desired circular trajectory, while keeping vehicle formation using a rigid graph theory. ${ }^{3)}$ Chen et al. proposed a tangent-plus-Lyapunov vector field guidance to find a short path and track ground targets for fixed-wing UAVs. ${ }^{4)}$

Other guidance logics were also developed and applied to the target observation or standoff tracking mission. Rysdyk introduced "good helmsman" behavior to design a guidance law for the target observation. ${ }^{5)}$ Prevost et al. proposed receding horizon model predictive control (MPC) for tracking ground targets using fixed-wing UAVs. ${ }^{6}$ Kim et al. proposed a nonlinear MPC framework for cooperative standoff tracking of a ground vehicle for fixed-wing UAVs. ${ }^{7}$

On the other hand, distance error dynamics can also be utilized to design a guidance law for the stationary target observation. ${ }^{8,9)}$ The lateral acceleration command is generated using the distance error and line-of-sight (LOS) angle information. A distance error dynamics-based guidance law is easy to understand physically and to implement to the UAV, since the error dynamics has a simple structure of the second-order mechanical system. However, the analysis for selecting the guidance gains was not systematic.

This paper deals with the stationary target observation of multiple UAVs. The nonlinear guidance law based on the distance error dynamics is analyzed to determine the UAV 
turning direction and to avoid invading the observation radius. It is shown that the turning direction is related to the LOS angular acceleration. Using this relationship, a suitable guideline for selecting gains of the nonlinear guidance law is presented taking into consideration the UAV turning direction.

This paper is organized as follows. In section 2, the distance error dynamics is formulated to derive the distance error dynamics-based guidance law. The relationship between the guidance gains and the turning direction of the UAV is analyzed, and a suitable guideline for choosing gains is summarized in section 3. An output feedback controller based on a linear-quadratic regulator (LQR) is used to design a stability augmentation system and autopilots for a linear six degree-of-freedom (DOF) UAV model in section 4. Numerical simulations for a point mass UAV model and a linear six DOF UAV model are presented to verify the performance of the proposed guidance law in section 5. Finally, conclusions are made in section 6 .

\section{Distance Error Dynamics-Based Guidance Law}

Let us consider the point mass UAV model shown in Fig. 1.

To formulate the kinematic equations, the following assumptions are required. First, the autopilot and seeker/ sensor dynamics are assumed to be much faster than the UAV dynamics so that they can be ignored. Second, the velocity of the UAV is assumed to be constant. Third, the angle-of-attack (AOA) of a UAV is small enough to be omitted. Fourth, the acceleration vector of the UAV is assumed to be perpendicular to the velocity vector. Then, the kinematic equations can be represented as follows

$$
\begin{aligned}
\dot{R} & =-V_{M} \cos \left(\sigma-\theta_{M}\right) \\
\dot{\sigma} & =\frac{V_{M}}{R} \sin \left(\sigma-\theta_{M}\right) \\
\dot{\theta}_{M} & =a_{M} / V_{M}=a_{c}
\end{aligned}
$$

Let us define a distance error as

$$
e_{R}=R-R_{d}
$$

where $R_{d}$ is the desired constant observation radius in the

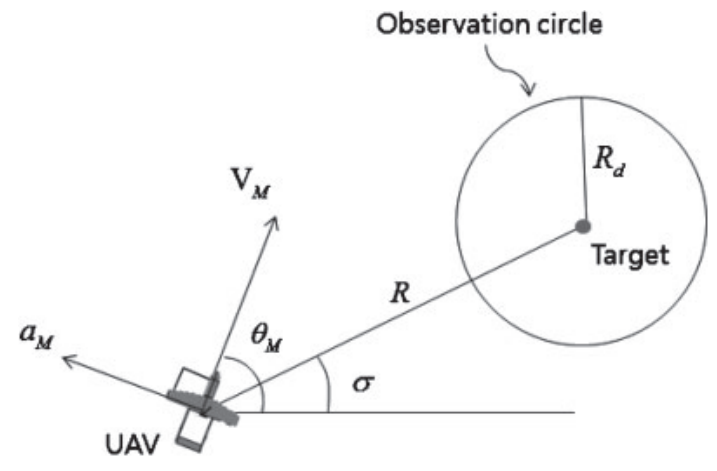

Fig. 1. Point mass model UAV geometry. given mission. Using the kinematic equations, the second time derivative of the distance error can be rewritten as

$$
\ddot{e}_{R}=V_{M} \sin \left(\sigma-\theta_{M}\right)\left(\dot{\sigma}-\dot{\theta}_{M}\right)=R \dot{\sigma}^{2}-R \dot{\sigma} a_{c}
$$

Let us propose a centrifugal acceleration command normalized by the UAV's velocity as

$$
a_{c}=\frac{1}{R \dot{\sigma}}\left(k_{1} \dot{e}_{R}+k_{2} e_{R}+R \dot{\sigma}^{2}\right)
$$

Substituting the proposed normalized lateral acceleration command into Eq. (5), the distance error dynamics can be represented as

$$
\ddot{e}_{R}+k_{1} \dot{e}_{R}+k_{2} e_{R}=0
$$

The distance error dynamics, Eq. (7), is represented as the second-order mechanical system. Therefore, the guidance gains can be defined as

$$
k_{1} \equiv 2 \zeta \omega_{n}, \quad k_{2} \equiv \omega_{n}^{2}
$$

Let us consider that $\zeta$ is larger than 1. This assumption is reasonable for a UAV carrying out the stationary target observation, since the UAV approaches the stationary target asymptotically and keeps the desired observation radius.

Using Eq. (8), the solution of the distance error equation can be obtained as

$$
e_{R}(t)=c_{1} e^{-\left(\zeta \omega_{n}+\omega_{n} \sqrt{\zeta^{2}-1}\right) t}+c_{2} e^{-\left(\zeta \omega_{n}-\omega_{n} \sqrt{\zeta^{2}-1}\right) t}
$$

In Eq. (9), $\zeta \omega_{n}-\omega_{n} \sqrt{\zeta^{2}-1}$ is always positive, because the damping ratio is larger than 1 . This means that the distance error converges to zero exponentially, as time passes. Therefore, straight flight paths will not be generated during the mission when the proposed guidance law is used.

\section{Gain Selection and Turning Direction Determina- tion}

Guidance gains should be carefully selected to accomplish the stationary target observation mission without invading the observation circle and colliding with other UAVs. In this study, it is assumed that obstacles do not exist, UAVs fly at the same altitude, and the UAVs start to fly to the target at a considerable distance from the circle.

Let us propose the guideline for choosing suitable guidance gains to determine the turning direction of each UAV and to maintain the turning direction of each UAV during the mission.

To avoid invading the desired observation radius, the distance error should be semi-positive during the mission. Then, the following theorem can be obtained.

Theorem 1. Distance error is semi-positive, when $c_{2}$ in Eq. (9) is positive and $\zeta$ in Eq. (8) is larger than 1.

Proof. The initial distance between the UAV and the stationary target is represented as

$$
R_{0}=c_{1}+c_{2}+R_{d}
$$

The initial distance rate is represented as 


$$
\begin{aligned}
\dot{R}_{0}= & -V_{M} \cos \left(\sigma_{0}-\theta_{M_{0}}\right) \\
= & -c_{1}\left(\zeta \omega_{n}+\omega_{n} \sqrt{\zeta^{2}-1}\right) \\
& -c_{2}\left(\zeta \omega_{n}-\omega_{n} \sqrt{\zeta^{2}-1}\right)
\end{aligned}
$$

Then, two coefficients can be obtained as

$$
\begin{gathered}
c_{1}=-\frac{\dot{R}_{0}+e_{R_{0}}\left(\zeta \omega_{n}-\omega_{n} \sqrt{\zeta^{2}-1}\right)}{2 \omega_{n} \sqrt{\zeta^{2}-1}} \\
c_{2}=\frac{\dot{R}_{0}+e_{R_{0}}\left(\zeta \omega_{n}+\omega_{n} \sqrt{\zeta^{2}-1}\right)}{2 \omega_{n} \sqrt{\zeta^{2}-1}}
\end{gathered}
$$

Let suppose that $c_{2}$ is positive.

i) $c_{1} \geq 0$

The distance error is semi-positive during the mission. This is because two coefficients are positive in this case.

ii) $c_{1}<0$

Subtraction of the absolute value of $c_{1}$ from $c_{2}$ gives

$$
c_{2}-\left|c_{1}\right|=e_{R_{0}}>0
$$

This means that the distance error is semi-positive, because $c_{2} e^{-\left(\zeta \omega_{n}-\omega_{n} \sqrt{\zeta^{2}-1}\right) t}$ is larger than $c_{1} e^{-\left(\zeta \omega_{n}+\omega_{n} \sqrt{\zeta^{2}-1}\right) t}$.

Q.E.D.

To satisfy the positive $c_{2}$ condition, the natural frequency should satisfy the following condition.

$$
\omega_{n_{c_{2}}}<\omega_{n}
$$

In the above condition, the lower bound of the natural frequency is defined as

$$
\omega_{n_{c_{2}}} \equiv \frac{-\dot{R}_{0}}{e_{R_{0}}\left(\zeta+\sqrt{\zeta^{2}-1}\right)}
$$

Now, the turning direction of each UAV should correspond with the turning direction of other UAVs. In this study, as aforementioned, the consensus of turning direction of each UAV is mainly focused, which simplifies the problem.

Let us define that the directions of LOS angle and heading angle are positive counter-clockwise as shown in Fig. 2. Then, the following proposition about the turning direction of the UAV can be obtained.
Proposition. When a UAV turns around the stationary target counter-clockwise, the LOS angle of the UAV increases. On the contrary, when a UAV turns around the target clockwise, the LOS angle of the UAV decreases.

Figure 3 shows the changes in the LOS angle during the UAV's clockwise turn. Using the above proposition, the theorem on the relationship between the LOS angular acceleration and the turning direction can be derived.

Theorem 2. If the LOS angular acceleration of a UAV can be controlled, then the turning direction of the UAV is determined as follows

$$
\begin{cases}\ddot{\sigma}>0 & \text { counter-clockwise } \\ \ddot{\sigma}<0 & \text { clockwise }\end{cases}
$$

Proof. When the LOS angular acceleration of a UAV is continuously positive, $\ddot{\sigma}>0$, although its initial LOS angular velocity was negative, $\dot{\sigma}_{0}<0$, the LOS angular velocity of the UAV becomes positive. This means that the LOS angle of the UAV increases, as time passes. Therefore, it can be stated that the UAV turns around the stationary target counter-clockwise. Likewise, if the LOS angular acceleration of a UAV is continuously negative, then finally, the UAV turns around the target clockwise.

$$
\left\{\begin{array}{l}
\text { counter-clockwise } \\
\ddot{\sigma}>0 \Rightarrow \dot{\sigma}>0 \text { as } t \rightarrow \infty \Rightarrow \sigma>0 \text { as } t \rightarrow \infty \\
\text { clockwise } \\
\ddot{\sigma}<0 \Rightarrow \dot{\sigma}<0 \text { as } t \rightarrow \infty \Rightarrow \sigma<0 \text { as } t \rightarrow \infty
\end{array}\right.
$$

Q.E.D.

Using Theorems 1 and 2, the guidance gains can be determined by considering the turning direction of a UAV for the stationary target observation. Let us provide a guideline for selecting suitable guidance gains of the distance error dynamics-based guidance. To provide the guideline, the following four cases are considered.

Case 1. Counter-clockwise turn and positive LOS angular velocity

According to Theorem 2, the LOS angular acceleration should be positive for a counter-clockwise turn. The LOS angular acceleration is represented as

$$
\ddot{\sigma}=\frac{\dot{R}}{R^{2} \dot{\sigma}}\left(2 \zeta \omega_{n} \dot{e}_{R}+\omega_{n}^{2} e_{R}-R \dot{\sigma}^{2}\right)
$$

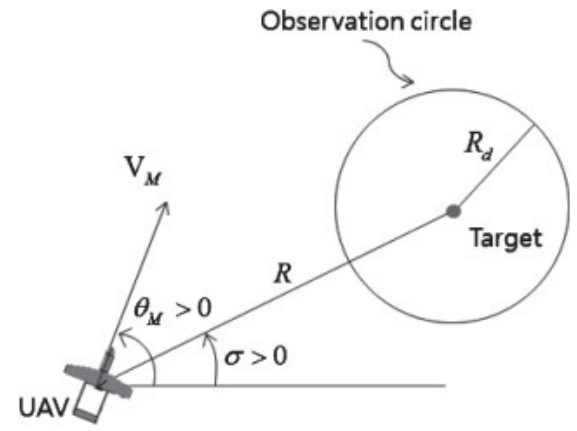

Fig. 2. Directions of LOS angle and heading angle.

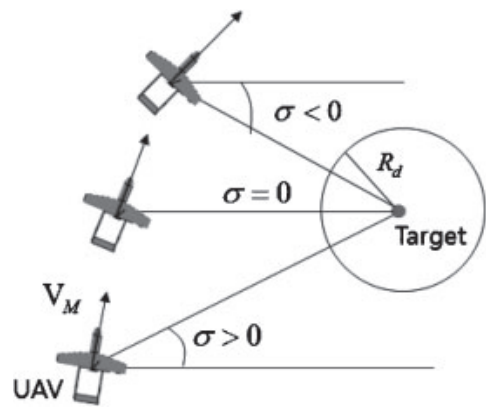

Fig. 3. Relationship between LOS angle change and turning direction. 
Therefore, $2 \zeta \omega_{n} \dot{e}_{R}+\omega_{n}^{2} e_{R}-R \dot{\sigma}^{2}$ should be negative in Case 1. This is because $\dot{R}$ is always negative during the mission. Then, the natural frequency should satisfy the following condition.

$$
0<\omega_{n}<\omega_{n_{l}}
$$

where

$$
\omega_{n_{l}}=\frac{-\zeta \dot{R}+\sqrt{\zeta^{2} \dot{R}^{2}+R \dot{\sigma}^{2} e_{R}}}{e_{R}}
$$

Additionally, the UAV should not invade the desired observation radius during the mission. Thus, the final condition of the natural frequency in Case 1 can be represented as

$$
\omega_{n_{c_{2}}}<\omega_{n}<\omega_{n_{l}}
$$

Corollary 1. The natural frequency $\omega_{n_{l}}$ always increases during the mission, as time passes.

Proof. Let us consider that the natural frequency is constant during the mission. The natural frequency $\omega_{n_{l}}$ can be divided into two terms as follows.

$$
\omega_{n_{l}}=\frac{-\zeta \dot{R}}{e_{R}}+\frac{\sqrt{\zeta^{2} \dot{R}^{2}+R \dot{\sigma}^{2} e_{R}}}{e_{R}}=\rho_{1}+\rho_{2}
$$

Then,

$$
\begin{aligned}
\lim _{t \rightarrow \infty} \rho_{1} & =\lim _{t \rightarrow \infty} \frac{-\zeta \dot{R}}{e_{R}} \\
& =\lim _{t \rightarrow \infty} \frac{\zeta\left[c_{1} \omega_{d_{1}} e^{-\omega_{d_{1}} t}+c_{2} \omega_{d_{2}} e^{-\omega_{d_{2}} t}\right]}{c_{1} e^{-\omega_{d_{1}} t}+c_{2} e^{-\omega_{d_{2}} t}} \\
& =\lim _{t \rightarrow \infty} \frac{\zeta\left[c_{1} \omega_{d_{1}} e^{-\left(\omega_{d_{1}}-\omega_{d_{2}}\right) t}+c_{2} \omega_{d_{2}}\right]}{c_{1} e^{-\left(\omega_{d_{1}}-\omega_{d_{2}}\right) t}+c_{2}} \\
& =\zeta \omega_{d_{2}} \\
& >0
\end{aligned}
$$

where

$$
\begin{aligned}
& \omega_{d_{1}}=\zeta \omega_{n}+\omega_{n} \sqrt{\zeta^{2}-1} \\
& \omega_{d_{2}}=\zeta \omega_{n}-\omega_{n} \sqrt{\zeta^{2}-1}
\end{aligned}
$$

Note that $\omega_{d_{1}}$ is larger than $\omega_{d_{2}}$.

Similarly, let us substitute Eq. (9) into $\rho_{2}$. Then,

$$
\begin{aligned}
\lim _{t \rightarrow \infty} \rho_{2} & =\lim _{t \rightarrow \infty} \frac{\sqrt{\zeta^{2} \dot{R}^{2}+R \dot{\sigma}^{2} e_{R}}}{e_{R}} \\
& =\lim _{t \rightarrow \infty} \frac{\sqrt{\zeta^{2} \dot{R}^{2}+e_{R}^{2} \dot{\sigma}^{2}+e_{R} \dot{\sigma}^{2} R_{d}}}{e_{R}} \\
& =\infty
\end{aligned}
$$

Therefore,

$$
\begin{aligned}
\lim _{t \rightarrow \infty} \omega_{n_{l}} & =\lim _{t \rightarrow \infty}\left(\rho_{1}+\rho_{2}\right) \\
& =\zeta \omega_{d_{2}}+\infty \\
\therefore \lim _{t \rightarrow \infty} \omega_{n_{l}} & =\infty
\end{aligned}
$$

It can be stated that $\omega_{n_{l}}$ always increases during the mission, as time passes.

Q.E.D.

Corollary 2. The natural frequency $\omega_{n_{c 2}}$ is always smaller than $\omega_{n_{l}}$, when $\zeta$ is larger than 1 .
Proof. Let us assume that the initial value of $\omega_{n_{l}}$ is smaller than $\omega_{n_{c_{2}}}$. Then,

$$
\frac{-\zeta \dot{R}_{0}+\sqrt{\zeta^{2} \dot{R}_{0}^{2}+R_{0} \dot{\sigma}_{0}^{2} e_{R_{0}}}}{e_{R_{0}}}<\frac{-\dot{R}_{0}}{e_{R_{0}}\left(\zeta+\sqrt{\zeta^{2}-1}\right)}
$$

The initial distance error is positive, and therefore

$$
\begin{aligned}
& -\zeta \dot{R}_{0}+\sqrt{\zeta^{2} \dot{R}_{0}^{2}+R_{0} \dot{\sigma}_{0}^{2} e_{R_{0}}}<\frac{-\dot{R}_{0}}{\zeta+\sqrt{\zeta^{2}-1}} \\
& -\zeta \dot{R}_{0}<\frac{-\dot{R}_{0}}{\zeta+\sqrt{\zeta^{2}-1}}
\end{aligned}
$$

The following conditions are always satisfied, because $\zeta$ is larger than 1.

$$
\begin{aligned}
& \zeta\left(\zeta+\sqrt{\zeta^{2}-1}\right)>1 \\
& \zeta>\frac{1}{\zeta+\sqrt{\zeta^{2}-1}}
\end{aligned}
$$

Note that $\dot{R}_{0}$ is always negative. Thus, we have

$$
-\zeta \dot{R}_{0}>\frac{-\dot{R}_{0}}{\zeta+\sqrt{\zeta^{2}-1}}
$$

However, Eq. (31) is a contradiction to Eq. (28). Therefore, the initial value of $\omega_{n_{l}}$ is larger than $\omega_{n_{c_{2}}}$. This means that $\omega_{n_{l}}$ is always larger than $\omega_{n_{c_{2}}}$ during the mission, due to Corollary 1.

Q.E.D.

Case 2. Counter-clockwise turn and negative LOS angular velocity

The term $2 \zeta \omega_{n} \dot{e}_{R}+\omega_{n}^{2} e_{R}-R \dot{\sigma}^{2}$ should be positive in Case 2, because $\dot{\sigma}$ is negative. Then, the natural frequency should satisfy the following condition.

$$
\omega_{n_{l}}<\omega_{n}
$$

Case 3. Clockwise turn and positive LOS angular velocity

According to Theorem 2, the term $2 \zeta \omega_{n} \dot{e}_{R}+\omega_{n}^{2} e_{R}-R \dot{\sigma}^{2}$ should be positive in Case 3 . Therefore, the natural frequency should satisfy condition (32).

Case 4. Clockwise turn and negative LOS angular velocity

According to Theorem 2, the term $2 \zeta \omega_{n} \dot{e}_{R}+\omega_{n}^{2} e_{R}-R \dot{\sigma}^{2}$ should be negative in Case 4 . Therefore, the natural frequency should satisfy condition (19).

Case 2 and Case 3 reflect that the heading angle of a UAV does not correspond with the desired turning direction, while Case 1 and Case 4 reflect that the heading angle of a UAV corresponds with the desired turning direction. However, if the natural frequency is chosen to satisfy Eq. (32) in Case 2 and Case 3, the heading angle of the UAV will be finally changed to meet the desired turning direction. In other words, this means that the situation, Case 2 or Case 3, can occur at the initial stage of the mission, because of sensor noise or the opposite initial heading angle of the UAV.

Now, let us propose a guideline to select proper guidance gains for performing the stationary target observation. 
First, the initial heading angle of a UAV is determined to correspond with its turning direction. If the desired turning direction of a UAV is counter-clockwise, then the initial heading angle of the UAV is selected as the value which is smaller than the LOS angle of the UAV. On the other hand, if the desired turning direction of a UAV is clockwise, then the initial heading angle of the UAV is selected as the value which is larger than the LOS angle of the UAV. This step is important to avoid the instantaneous singularity.

Then, the natural frequency is determined to satisfy condition (33). Although the initial heading angle of the UAV is determined to correspond with the desired turning direction, the UAV can fly along the opposite turning direction initially due to the sensor noise. In this case, the natural frequency should be determined to satisfy condition (34) until the UAV flies along the desired turning direction. At the switching step, from the situation using the natural frequency (34) to the situation using the natural frequency (33), the instantaneous singularity can occur. This step may occur at the early stage during the mission, because it is assumed that the UAV starts to fly considerably distant from the circle. Therefore, the limit of the guidance command should be determined considering the dynamics of the UAV. Note that the damping ratio should be larger than 1 during the mission.

$$
\omega_{n}=\mu_{1} \omega_{n_{l_{0}}}
$$

where

$$
\begin{aligned}
& \frac{\omega_{n_{c_{2}}}}{\omega_{n_{l_{0}}}}<\mu_{1}<1 . \\
& \omega_{n}=\omega_{n_{l}}+\mu_{2}
\end{aligned}
$$

where

$$
\mu_{2}>0 \text {. }
$$

Figure 4 shows the relationship between the initial heading angle of the UAV and its turning direction. Figure 5 summarizes the guideline of choosing the proper guidance gains.

\section{Control Law}

The proposed guidance law is associated with the roll angle command. To apply the guidance law to the linear six DOF UAV model, the longitudinal and lateral controllers are designed using the output feedback controller based on LQR. ${ }^{10)}$

The longitudinal distance error, $d$, is defined as the difference between the actual flight path and the reference flight path. The output feedback controller based on LQR is designed to track the flight path angle command while maintaining the zero longitudinal distance error. The dynamics of the longitudinal distance error is represented as

$$
\dot{d}=V_{M} \sin \left(\gamma-\gamma_{\mathrm{ref}}\right) \approx V_{M}\left(\gamma-\gamma_{\mathrm{ref}}\right)
$$

The longitudinal tracking errors are defined as

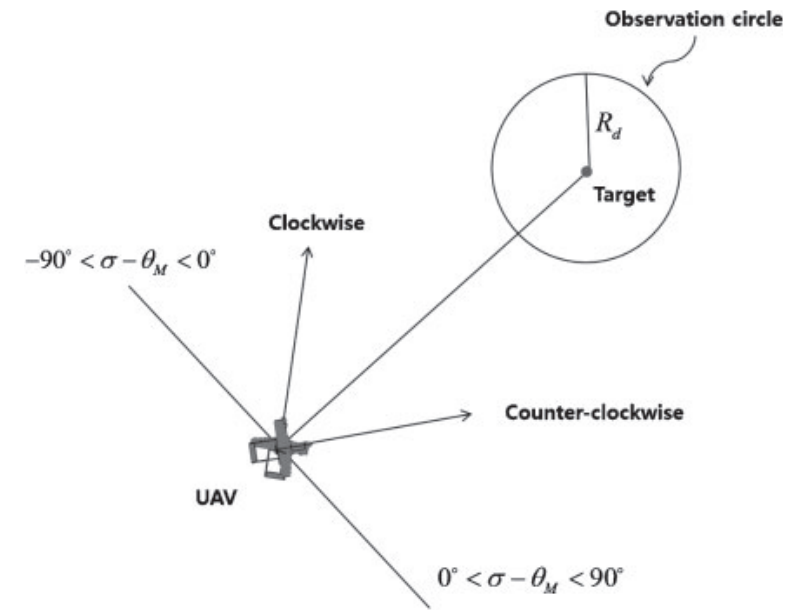

Fig. 4. Geometry of initial heading angle and turning direction.

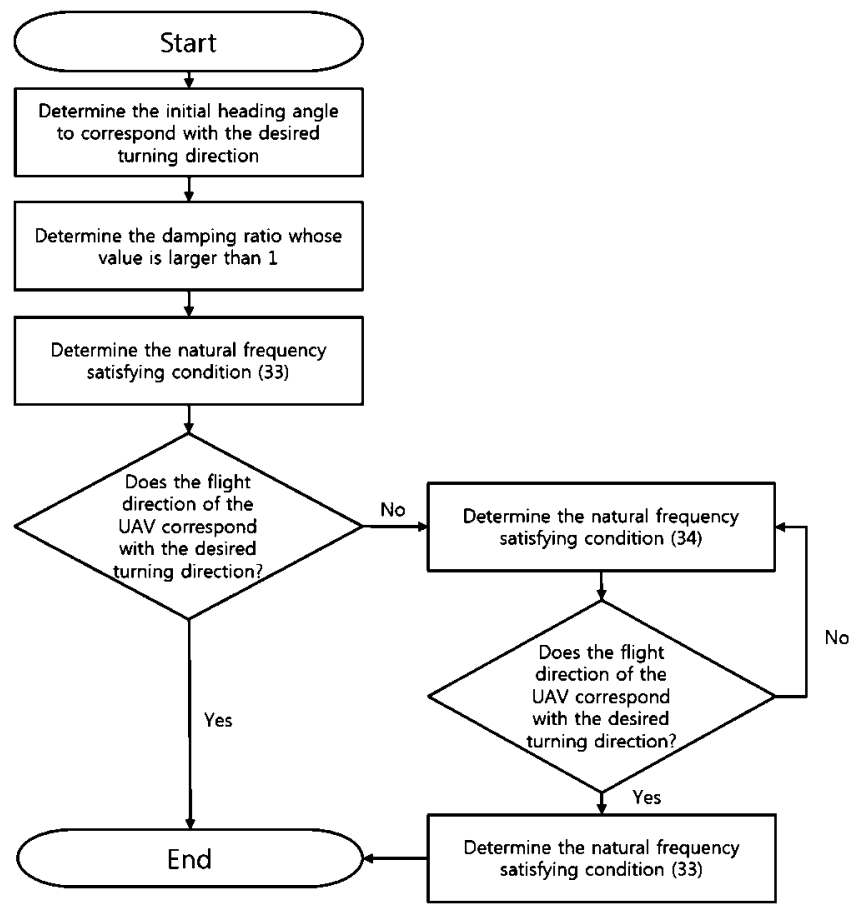

Fig. 5. Flow chart choosing guidance gains.

$$
\begin{gathered}
e_{V}=V_{\mathrm{ref}}-V_{M} \\
e_{d}=d_{\mathrm{ref}}-d
\end{gathered}
$$

A lateral controller is also designed to track the roll angle command, $\phi_{\text {ref }}$, while maintaining the zero washed-out yaw rate, $r_{w}$. The lateral tracking errors are defined as

$$
\begin{aligned}
& e_{r}=r_{\text {ref }}-r_{w} \\
& e_{\phi}=\phi_{\text {ref }}-\phi
\end{aligned}
$$

Roll angle command, $\phi_{\text {ref }}$, can be obtained as

$$
\frac{a_{c} V_{M}}{g}=\tan \left(\phi_{\mathrm{ref}}\right)
$$

Control surface angles should be limited, although the acceleration command is limited. Note that $a_{c}$ is the centrifugal acceleration normalized by the velocity of the UAV, and therefore, the velocity of the UAV should be multiplied 


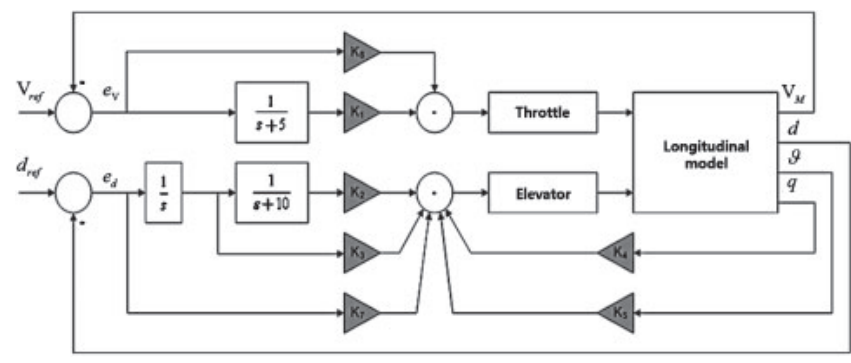

Fig. 6. Block diagram of longitudinal control law.

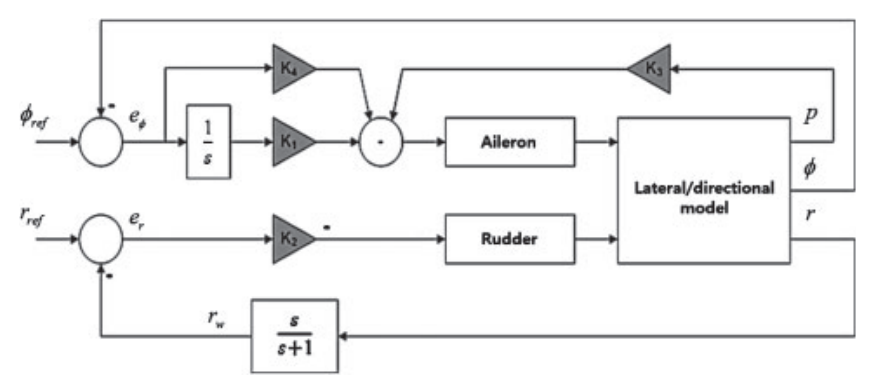

Fig. 7. Block diagram of lateral control law.

by the normalized acceleration to calculate the roll angle command. Longitudinal and lateral controller block diagrams are shown in Figs. 6 and 7.

The state vectors and control surface inputs vectors are defined as

$$
\begin{aligned}
\boldsymbol{x}_{\text {long }} & =\left[\begin{array}{llll}
V_{M} & \alpha & \vartheta & q
\end{array}\right]^{\mathrm{T}} \\
\boldsymbol{x}_{\text {lat }} & =\left[\begin{array}{llll}
\beta & \phi & p & r
\end{array}\right]^{\mathrm{T}} \\
\boldsymbol{u}_{\text {long }} & =\left[\begin{array}{ll}
\delta_{\text {thr }} & \delta_{\text {ele }}
\end{array}\right]^{\mathrm{T}} \\
\boldsymbol{u}_{\text {lat }} & =\left[\begin{array}{ll}
\delta_{\text {ail }} & \delta_{\text {rud }}
\end{array}\right]^{\mathrm{T}}
\end{aligned}
$$

where $\delta_{\text {thr }}, \delta_{\text {ele }}, \delta_{\text {ail }}$ and $\delta_{\text {rud }}$ are the thrust displacement, elevator displacement, aileron displacement and rudder displacement, respectively.

\section{Numerical Simulations}

Numerical simulations are performed to verify the performance of the proposed guidance law. Target observation by the coordinated turn with a desired radius is considered as the mission of the multiple UAVs. Simulation using the point mass UAV model is first performed to show the performance of the proposed guidance law. Then, simulation using the linear six DOF UAV model is done. No wind and no obstacle environments are considered in both simulations.

\subsection{Point mass UAV model simulation}

For the simulation, the desired observation radius is set as $300 \mathrm{~m}$. The initial position of each UAV is $(0,0)$, $(1,600,100)$ and $(300,1,600)$, respectively, and the position of the stationary target is $(800,600)$. The velocity of each $\mathrm{UAV}$ is $15 \mathrm{~m} / \mathrm{s}$. The damping ratio, $\zeta$, and gain design parameters, $\mu_{1}$ and $\mu_{2}$, are set as $1.2,0.8$ and 0.05 , respectively. The limit of the normalized acceleration command is chosen as $2 g / V_{M}$. In the counter-clockwise turn case,

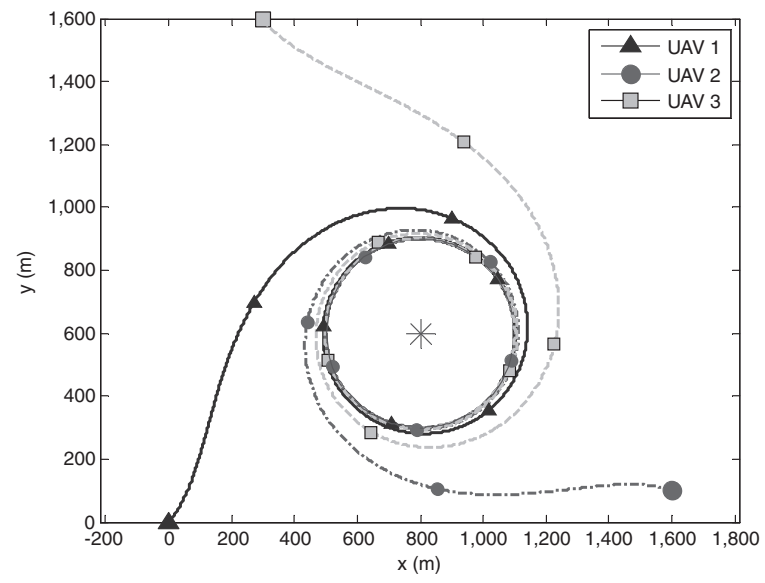

Fig. 8. 2D trajectories of point mass UAV models (clockwise turn).
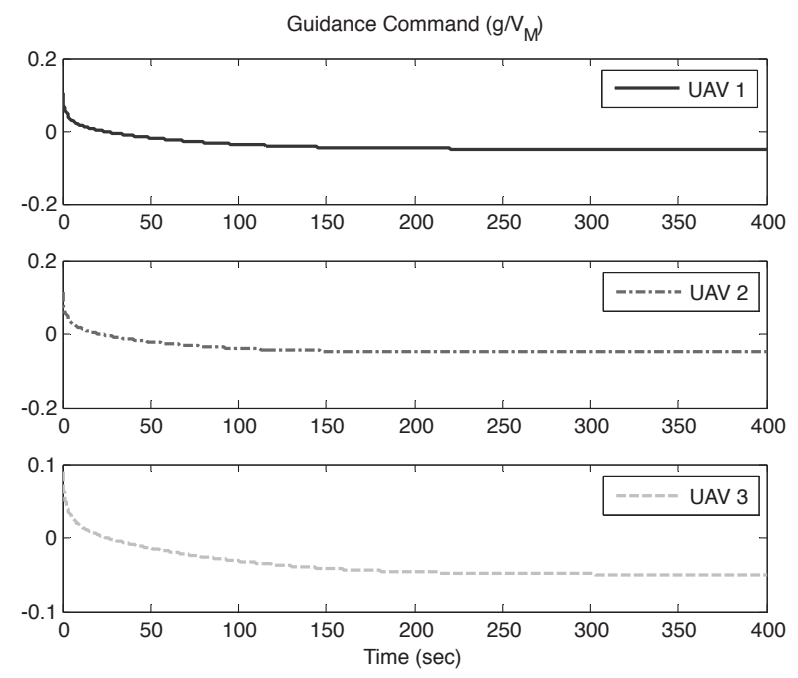

Fig. 9. Normalized guidance command of point mass UAV models (clockwise turn)

the initial heading angle of each UAV is set as 10 degrees smaller than its initial LOS angle. In the clockwise turn case, the initial heading angle of each UAV is set as 10 degrees larger than its initial LOS angle.

Figure 8 shows the two-dimensional trajectories of the point mass UAV model in the clockwise turn case while maintaining the desired observation radius. Figure 9 shows the normalized acceleration guidance command histories of the point mass UAV model in the clockwise turn. Although the initial normalized command of each point mass UAV has a positive value, the normalized command of each vehicle decreases and finally has a negative value to maintain the clockwise turning direction.

Figure 10 shows the two-dimensional trajectories of the point mass UAV model in the counter-clockwise turn case while maintaining the desired observation radius. Figure 11 shows the normalized acceleration guidance command histories of the point mass UAV models in the counterclockwise turn. Similarly, the normalized command of each vehicle increases and finally has positive value to maintain the counter-clockwise turning direction. 


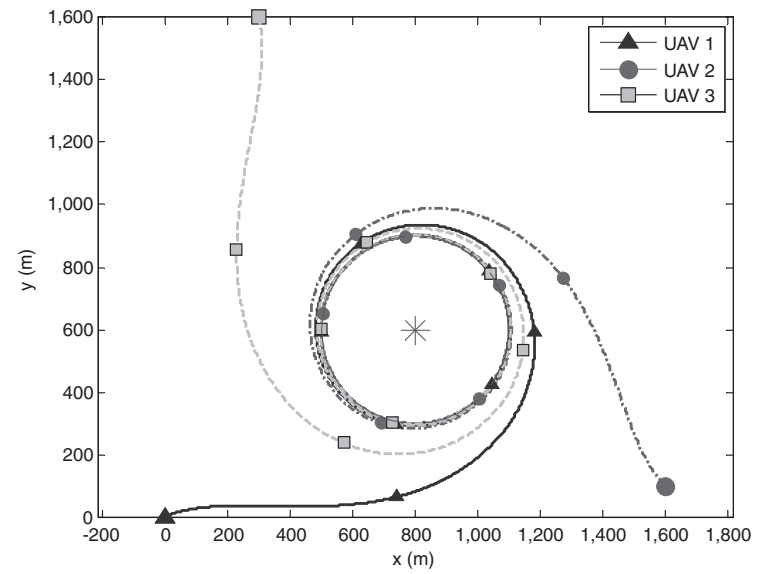

Fig. 10. 2D trajectories of point mass UAV models (counter-clockwise turn).
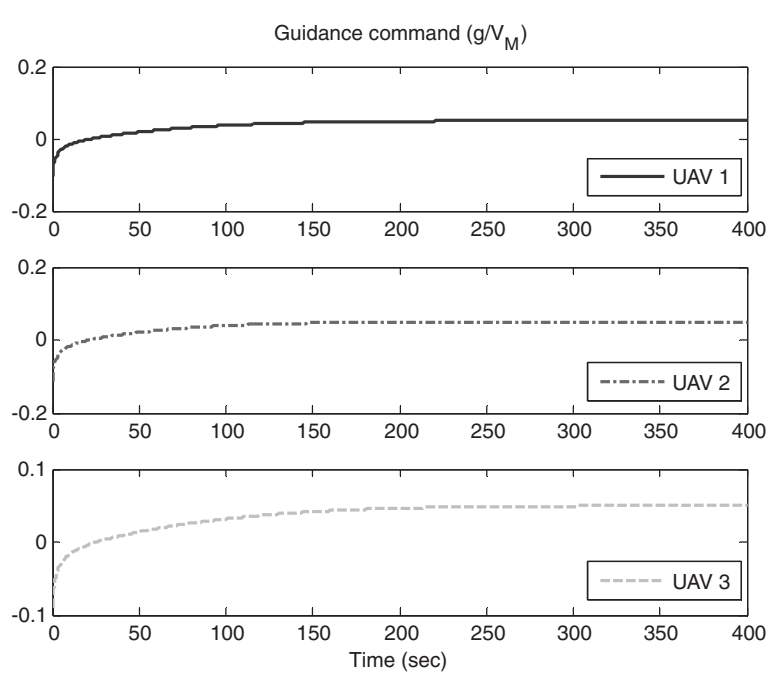

Fig. 11. Normalized guidance command of point mass UAV models (counter-clockwise turn).

\subsection{Six DOF UAV model simulation}

The guidance law is applied to a linear six DOF UAV model. The initial conditions and the selected gains are the same as those of the point mass UAV model simulation. The guidance command can be generated using only GPS position data. Therefore, it is important that GPS sensor noise is considered. In this study, it is assumed that GPS sensor noise has Gaussian distribution with zero mean and variance of 2.5. In addition, lowpass filters are considered to utilize noised GPS position data. In this simulation, the limit of the guidance command is set as $2 g / V_{M}$. The limits of displacement of the aileron and elevator control surfaces are set as \pm 20 degrees and \pm 10 degrees, respectively.

Figure 12 shows the two-dimensional trajectories of three six DOF UAV models in clockwise turn while maintaining the desired observation circle. Figure 13 shows the time history of the distance errors of three UAVs. Each distance error converges to zero, as shown in Fig. 13. UAV3 tries to turn around the stationary target counter-clockwise initially. After a few seconds, however, the flight direction of the $\mathrm{UAV} 3$ is corrected, due to the selected gains.

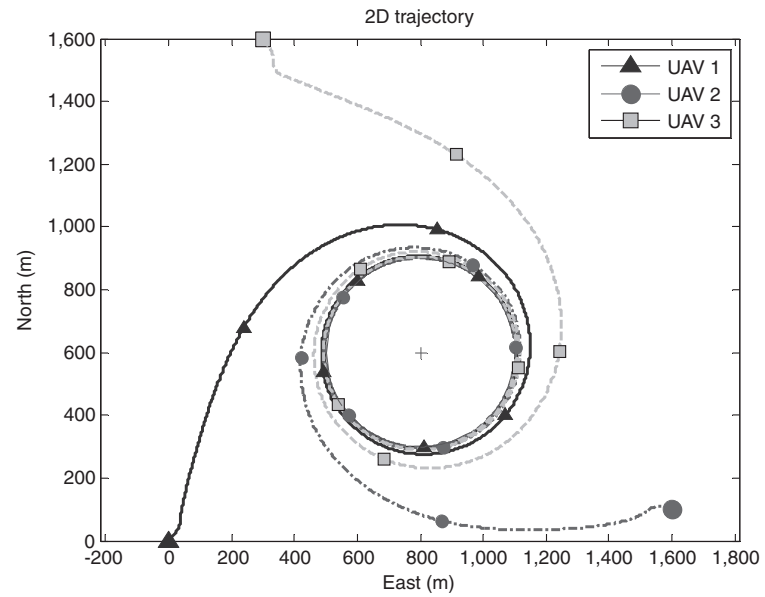

Fig. 12. 2D trajectories of three six DOF UAVs (clockwise turn).

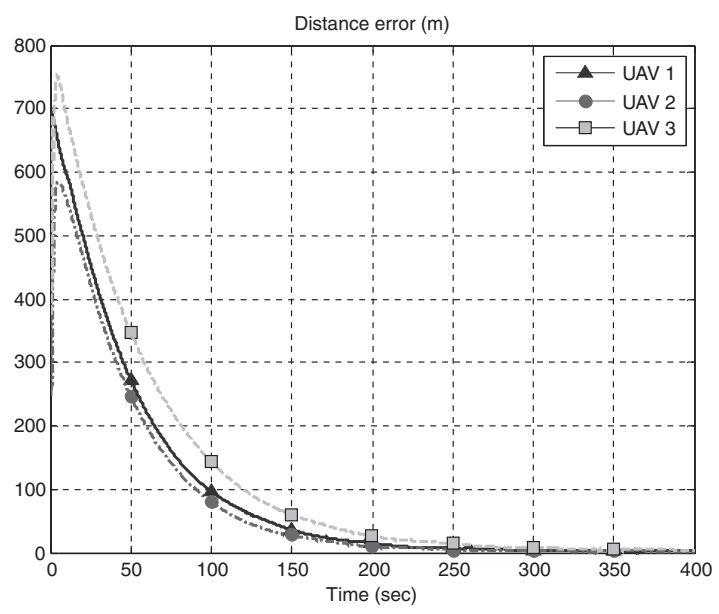

Fig. 13. Distance error histories of three six DOF UAVs (clockwise turn).
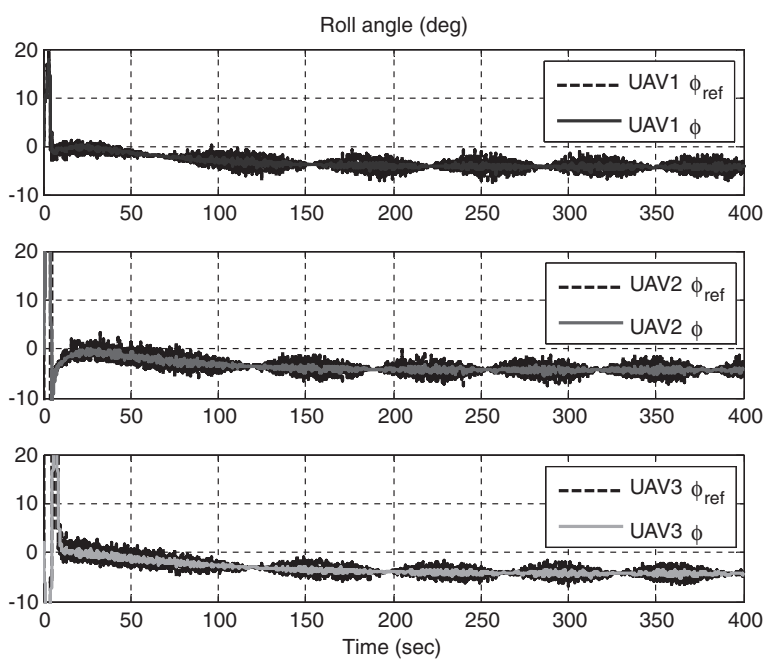

Fig. 14. Roll angle commands and actual roll angles of three six DOF UAVs (clockwise turn).

Figure 14 shows the time history of roll angle commands of the three UAVs. The dashed line denotes the roll angle commands of each UAV and the solid lines denote the actual roll angle of each UAV, respectively. Roll angle commands are chattering, due to GPS sensor noise. Figure 15 

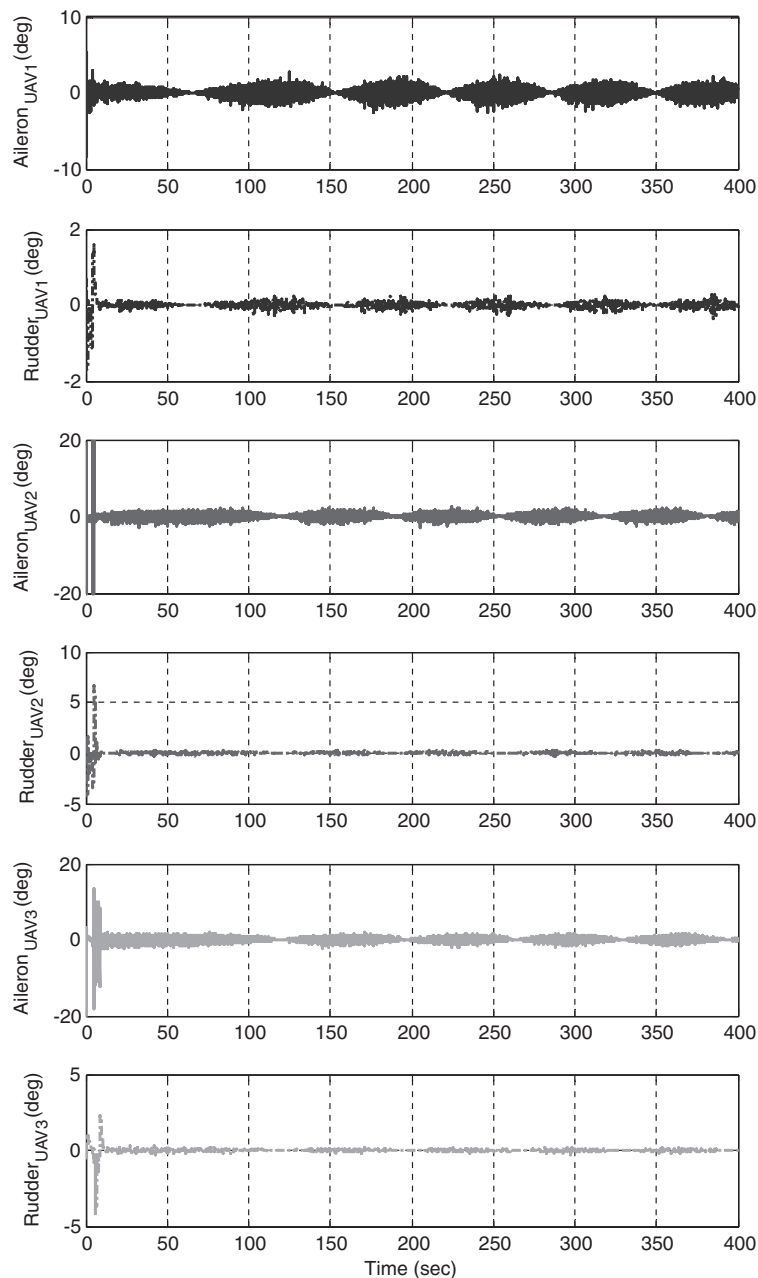

Fig. 15. Displacement of aileron and rudder control surfaces of three six DOF UAVs (clockwise turn).

shows the time history of the displacement of the aileron and rudder control surfaces. Displacement of the aileron and rudder control surfaces of each UAV is chattering, due to chattering roll angle commands. Although lowpass filters are used for the signal processing of the GPS data, the filtered position data still includes some noise. Moreover, unlike the ideal point mass simulation, the velocity of the UAV changes in the six DOF simulation. Therefore, the low frequency oscillation is observed in Figs. 14 and 15.

\section{Conclusions}

A nonlinear guidance law based on distance error dynamics was proposed and analyzed to perform the stationary target observation. The guideline for selecting suitable guid- ance gains was provided to determine the turning direction and to avoid invading the observation circle. The normalized centrifugal acceleration was used to reduce the distance error. An output feedback controller based on a LQR was utilized to design the lateral and longitudinal control systems. Numerical simulations using a point mass model and a linear six DOF UAV model were performed to verify the performance of the proposed guidance law. The implementation of the proposed guidance law and the guideline for choosing the gains were validated using the same gains in the point mass UAV model and a linear six DOF UAV model. Further work is required to consider the phase angle difference among UAVs for improving the mission capability.

\section{Acknowledgments}

This work was supported by the Defense Research Grant funded by the Agency for Defense Development under the contract UD120013JD.

\section{References}

1) Frew, E. W., Lawrence, D. A. and Morris, S.: Coordinated Standoff Tracking of Moving Target Using Lyapunov Guidance Vector Fields, J. Guid. Control Dynam., 31 (2008), pp. 290-306.

2) Lawrence, D. A., Frew, E. W. and Pisano, W. J.: Lyapunov Vector Fields for Autonomous Unmanned Aircraft Flight Control, J. Guid. Control Dynam., 31 (2008), pp. 1220-1229.

3) Summers, T. H., Akella, M. R. and Mears, M. J.: Coordinated Standoff Tracking of Moving Targets: Control Laws and Information Architectures, J. Guid. Control Dynam., 32 (2009), pp. 56-69.

4) Chen, H., Chang, K. C. and Agate, C. S.: Tracking with UAV using Tangent-plus-Lyapunov Vector Field Guidance, 12th International Conference on Information Fusion, Seattle, WA, 2009.

5) Rysdyk, R.: Unmanned Aerial Vehicle Path Following for Target Observation in Wind, J. Guid. Control Dynam., 29 (2006), pp. 10921100 .

6) Prevost, C. V., Theriault, O., Deshiens, A., Poulin, E. and Gagnon, E.: Receding Horizon Model-Based Predictive Control for Dynamic Target Tracking; a Comparative Study, AIAA Guidance, Navigation, and Control Conference, Chicago, IL, 2009.

7) Kim, S., Oh, H. and Tsourdos, A.: Nonlinear Model Predictive Coordinated Standoff Tracking of Moving Ground Vehicle, AIAA Guidance, Navigation, and Control Conference, Portland, OR, 2011.

8) Kim, M., Kim, Y. and Park, C. G.: Guidance Gain Analysis via Oscillation Motion of Error Dynamics-Based Guidance Law for Stationary Target Observation, 4th European Conference for Aerospace Sciences, St. Petersburg, Russia, 2011.

9) Kim, M. and Kim, Y.: Error Dynamics-Based Lyapunov Guidance Law for Stationary Target Observation, The 18th IFAC World Congress, Milano, Italy, 2011.

10) Stevens, B. L. and Lewis, F. L.: Aircraft Control and Simulation, 2nd ed., John Wiley \& Sons, NJ, 2003. 